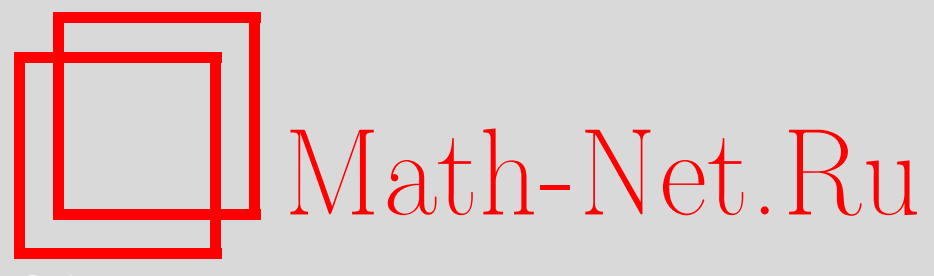

С. Е. Степанов, О некоторых конформных и проективных скалярных инвариантах риманова многообразия, Матем. заметки, 2006, том 80, выпуск 6, 902-907

DOI: https://doi.org/10.4213/mzm3365

Использование Общероссийского математического портала Math-Net.Ru подразумевает, что вы прочитали и согласны с пользовательским соглашением http://www . mathnet.ru/rus/agreement

Параметры загрузки:

IP : 54.197 .217 .227

26 апреля 2023 г., 16:10:23

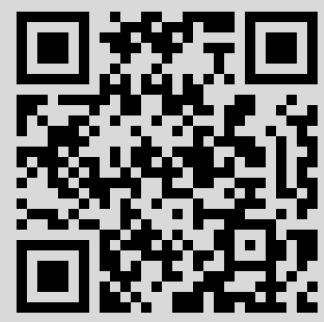




\section{О НЕКОТОРЫХ КОНФОРМНЫХ И ПРОЕКТИВНЫХ СКАЛЯРНЫХ ИНВАРИАНТАХ РИМАНОВА МНОГООБРАЗИЯ}

\section{C. Е. Степанов}

Доказывается, что на $n$-мерном замкнутом ориентированном римановом многообразии для всех $1 \leqslant r \leqslant n-1$ векторные пространства конформно-киллинговых, киллинговых и замкнутых конформно-киллинговых $r$-форм являются конечномерными, а их размерности - числа $t_{r}$ являются конформными, а $k_{r}$ и $p_{r}$ - проективными скалярными инвариантами многообразия, которые обладают следующими свойствами двойственности: $t_{r}=t_{n-r}$ и $k_{r}=p_{n-r}$. Кроме того, найден явный вид конформно-киллинговой $r$-формы на конформно плоском $n$-мерном римановом многообразии.

Библиография: 10 названий.

1. Введение. Полагаем $(M, g)$ римановым многообразием размерности $n \geqslant 2$. Для каждого целого $1 \leqslant r \leqslant n$ обозначим через $\Omega^{r}(M, \mathbb{R})$ векторное пространство дифференциальных $r$-форм на $(M, g)$ и рассмотрим его подпространства. Наиболее известным является подпространство гармонических $r$-борм $H^{r}(M, \mathbb{R})$, которое на замкнутом ориентированном римановом многообразии $(M, g)$ имеет конечную размерность $b_{r}$, носящую название $r$-го числа Бетти. Числа Бетти удовлетворят двойственности Пуанкаре $b_{r}=b_{n-r}$ и для $n=2 r$ конформно инвариантны (см. [1; c. 85]). Менее известны три других подпространства. Первое из них $-T^{r}(M, \mathbb{R})$ состоит из конформно-киллинговых дифференииальных $r$-форм или, иначе, конформных тензоров Киллинга-Яно ранга $r$, которые были введены на римановом многообразии $(M, g)$ как обобщение конформно-киллинговых векторных полей. Второе из них $-K^{r}(M, \mathbb{R})$ состоит из козамкнутых конформно-киллинговых дифференциальных $r$-форм, называемых еще киллинговыми $r$-формами или, по другой терминологии, тензорами Киллинга-Яно ранга $r$, которые служат обобщением векторных полей Киллинга. И, наконец, третье подпространство $P^{r}(M, \mathbb{R})$ состоит из замкнутых конформно киллинговых дифференциалъных $r$-форм или, по другой терминологии, замкнутых конформно киллинговых тензоров ранга $r$, которые являются обобщением конциркулярных векторных полей (см. обзор [1]).

Перечисленные три векторные подпространства на замкнутом ориентированном римановом многообразии $(M, g)$ обладают свойствами, аналогичными свойствам подпространства гармонических форм.

ТЕОРема 1. На п-мерном замкнутом ориентированном римановом многообразии $(M, g)$ для всех $1 \leqslant r \leqslant n-1$ векторные пространства $T^{r}(M, \mathbb{R}), K^{r}(M, \mathbb{R}) u$ $P^{r}(M, \mathbb{R})$ являются конечномерными, а их размерности - числа $t_{r}=\operatorname{dim} T^{r}(M, \mathbb{R})$

(C) C. Е. Степанов, 2006 
являются конформными, $k_{r}=\operatorname{dim} K^{r}(M, \mathbb{R})$ u $p_{r}=\operatorname{dim} P^{r}(M, \mathbb{R})$ - проективными скалярными инвариантами многообразия, которые обладают следующими свойствами двойственности: $t_{r}=t_{n-r} u k_{r}=p_{n-r}$.

Заметим, что в отличии от $t_{r}, k_{r}$ и $p_{r}$ числа Бетти $b_{r}$ являются еще и топологическими инвариантами многообразия $(M, g)$.

Используя проективную инвариантность козамкнутых и замкнутых конформно киллинговых форм, в работах [2] и [3] мы получили их явное выражение в локальной системе координат на проективно плоском псевдоримановом многообразии. Это позволило выписать интегралы специальных уравнений Максвелла и операторы симметрии безмассовых уравнений Дирака на данном многообразии.

В настоящей статье, опираясь на конформную инвариантность конформно киллинговых форм, мы получим их явное выражение в локальной системе координат на конформно плоском многообразии, что, в частности, позволит выписать операторы симметрии уравнений Дирака при наличии массы (см. [3]) на конформно плоском псевдоримановом многообразии.

ТЕорема 2. На п-мерном конформно плоском римановом многообразии $(M, g)$ каждая точка обладает окрестностъю с локальной системой координат $x^{1}, \ldots, x^{n}$, в которой произвольная конформно киллинговая $r$-форма $\omega, 1 \leqslant r \leqslant n-1$, имеет локальные компоненты

$$
\omega_{i_{1} \ldots i_{r}}=e^{-(r+1) \sigma}\left(A_{k j i_{1} \ldots i_{r}} x^{k} x^{j}+B_{j i_{1} \ldots i_{r}} x^{j}+C_{i_{1} \ldots i_{r}}\right) .
$$

При этом стоящие в правых частях равенств (1) коэфбициенты $A_{k j i_{1} \ldots i_{r}}, B_{i_{1} \ldots i_{r}} u$ $C_{i_{1} \ldots i_{r}}$ являются постоянными, кососимметричными по индексам $i_{1}, \ldots, i_{r}$ и подчиняются следующим условиям:

$$
\begin{gathered}
A_{k j i i_{2} \ldots i_{r}}+A_{k i j i_{2} \ldots i_{r}}=2 A_{k i_{2} \ldots i_{r}} \bar{g}_{i j}-\sum_{a=2}^{r}(-1)^{a}\left(A_{k i i_{2} \ldots \hat{i}_{a} \ldots i_{r}} \bar{g}_{j i_{a}}+A_{k j i_{2} \ldots \hat{i}_{a} \ldots i_{r}} \bar{g}_{j i_{a}}\right), \\
B_{j i i_{2} \ldots i_{r}}+B_{i j i_{2} \ldots i_{r}}=2 B_{i_{2} \ldots i_{r}} \bar{g}_{i j}-\sum_{a=2}^{r}(-1)^{a}\left(B_{i i_{2} \ldots \hat{i}_{a} \ldots i_{r}} \bar{g}_{j i_{a}}+B_{j i_{2} \ldots \hat{i}_{a} \ldots i_{r}} \bar{g}_{j i_{a}}\right)
\end{gathered}
$$

$\partial \Omega я$

$$
A_{k i_{2} \ldots i_{r}}=\frac{1}{n-p+1} \bar{g}^{j l} A_{k j l i_{2} \ldots i_{r}}, \quad B_{i_{2} \ldots i_{r}}=\frac{1}{n-p+1} \bar{g}^{j l} B_{j l i_{2} \ldots i_{r}}
$$

и постоянных компонент $\bar{g}_{i j}$ метрического тензора $\bar{g}$ такого, что $g=e^{-2 \sigma} \bar{g}$.

2. Доказательство утверждений. Для произвольного гладкого отображения $f:(M, g) \rightarrow(\bar{M}, \bar{g})$ римановых многообразий обозначим через $f_{*}$ его дифференциал, а через $f^{*}$ - транспонированное к $f_{*}$ отображение. Тогда для любой дифференциальной $r$-формы $\bar{\omega}$ на $(\bar{M}, \bar{g})$ можно определить дифференциальную $r$-форму $\omega:=f^{*} \bar{\omega}$ на многообразии $(M, g)$. Справедлива следующая

Лемма 1. Пусть $f:(M, g) \rightarrow(\bar{M}, \bar{g})$ - конформныц диффеоморфизм $n$-мерных римановых многообразий, определяемый условием $f^{*} \bar{g}=e^{2 \sigma} g, u \omega-$ конформно киллинговая $r$-форма, $1 \leqslant r \leqslant n-1$, на многообразии $(M, g)$. Тогда $r$-форма $\widetilde{\omega}$ на многообразии $(\bar{M}, \bar{g})$ такая, что $f^{*} \widetilde{\omega}=e^{(r+1) \sigma} \omega$, будет конформно киллинговой. 
ДокАЗАТЕЛЬСтво. Пусть $f:(M, g) \rightarrow(\bar{M}, \bar{g})$ - диффеоморфизм. Тогда (см. [4; c. 67]) для любой пары точек $x \in M$ и $\bar{x}=f(x) \in \bar{M}$ можно выбрать такие карты $(U, \varphi)$ для $x \in U$ и $(\bar{U}, \bar{\varphi})$ для $\bar{x} \in \bar{U}$, что диффеоморфизм $f$ в этих картах задается уравнениями вида $\bar{x}^{1}=x^{1}, \ldots, \bar{x}^{n}=x^{n}$. Будем говорить, что многообразия $(M, g)$ и $(\bar{M}, \bar{g})$ отнесены к общей по отношению к данному диффеоморфизму $f$ системе локальных координат $x^{1}, \ldots, x^{n}$. В такой системе локальных координат конформный диффеоморфизм $f:(M, g) \rightarrow(\bar{M}, \bar{g})$ определяется условием $\bar{g}=e^{2 \sigma} g$, из которого в качестве следствий выводятся равенства (см. [1; с. 84-85])

$$
\begin{gathered}
\bar{\nabla}_{X} Y=\nabla_{X} Y+d \sigma(X) Y+d \sigma(Y) X-g(X, Y) \operatorname{grad} \sigma, \\
\bar{d}^{*} \omega=e^{-2 \sigma}\left\{d^{*} \omega-(n-2 r) i_{\operatorname{grad}} \omega\right\}
\end{gathered}
$$

для связностей Леви-Чивиты $\nabla$ и $\bar{\nabla}$ и операторов кодифференцирования $d^{*}$ и $\bar{d}^{*}$ римановых многообразий $(M, g)$ и $(\bar{M}, \bar{g})$ соответственно, произвольных векторных полей $X=X^{i} \partial / \partial x^{i}$ и $Y=Y^{k} \partial / \partial x^{k}$ и внутреннего умножения $i_{\operatorname{grad} \sigma} \omega$, т.е. свертки $r$-формы $\omega$ с вектором $\operatorname{grad} \sigma$.

На многообразии $(M, g)$ конформно киллинговы $r$-формы составляют ядро естественного дифференциального оператора первого порядка $D$, действие которого на произвольную форму $\omega \in C^{\infty} \Lambda^{r} M$ задается равенством

$$
D \omega=\nabla \omega-(r+1)^{-1} d \omega-(n-r+1)^{-1} g \wedge d^{*} \omega
$$

для оператора внешнего дифференцирования $d: C^{\infty} \Lambda^{r} M \rightarrow C^{\infty} \Lambda^{r+1} M$, упоминаемого выше сопряженного к $d$ оператора кодифференцирования $d^{*}: C^{\infty} \Lambda^{r} M \rightarrow$ $C^{\infty} \Lambda^{r-1} M$ и внешнего умножения $\wedge$ дифференциальной $(r-1)$-формы $d^{*} \omega$ на метрический тензор $g$, которая определяется по закону

$$
\left(g \wedge d^{*} \omega\right)\left(X_{0}, X_{1}, \ldots, X_{r}\right)=\sum_{a=2}^{r}(-1)^{a} g\left(X_{0}, X_{a}\right)\left(d^{*} \omega\right)\left(X_{1}, \ldots, \widehat{X}_{a}, \ldots, X_{r}\right)
$$

для произвольных $X_{0}, \ldots, X_{r} \in C^{\infty} T M$ (см. [2], [5], [6]). Тогда в общей по отношению к данному конформному диффеоморфизму $f$ системе локальных координат $x^{1}, \ldots, x^{n}$ связь между оператором $D$ и аналогичным ему оператором $\bar{D}$ на многообразии $(\bar{M}, \bar{g})$ в силу $(2)$ и (3) будет задаваться равенством

$$
\bar{D} \omega=D \omega-(r+1)\left\{d \sigma \otimes \omega-(r+1)^{-1} d \sigma \wedge \omega-(n-r+1)^{-1} g \wedge i_{\operatorname{grad} \sigma} \omega\right\},
$$

где $\bar{\omega}=\omega$ в выбранной системе координат. А потому для формы $\widetilde{\omega}=e^{(r+1) \sigma} \omega$ будет справедливым соотношение $\bar{D} \widetilde{\omega}=e^{(r+1) \sigma} D \omega$, которое и доказывает лемму, поскольку в этом случае

$$
\omega \in \operatorname{Ker} D \Longleftrightarrow \widetilde{\omega} \in \operatorname{Ker} \bar{D} .
$$

ДоказАТЕЛЬСтво тЕоРемы 1. В работе [6] на замкнутом ориентированном римановом многообразии $(M, g)$ был найден сопряженный к естественному оператоpy $D$ оператор $D^{*}$, построен грубый лапласиан $D^{*} D: C^{\infty} \Lambda^{p} M \rightarrow C^{\infty} \Lambda^{p} M$ и доказано, что $\operatorname{Ker} D^{*} D$ исчерпывается $r$-формами пространства $T^{r}(M, \mathbb{R})$. Операторы вида $D^{*} D$ являются эллиптическими (см., например, [7]) и, как следствие этого, ядро 
$\operatorname{Ker} D^{*} D$ каждого из них представляет собою конечномерное векторное пространство (см. [1; с. 631-632] и [8; гл. 11]). В частности, таковым будет и пространство $T^{r}(M, \mathbb{R})$, для которого полагаем $t_{r}=\operatorname{dim} T^{r}(M, \mathbb{R})($ см. также [1; с. 49]).

При конформном преобразовании метрики $\bar{g}=e^{2 \sigma} g$ конформно киллинговые $r$-формы $\omega$ многообразия $(M, g)$ зададут конформно киллинговые $r$-формы $\bar{\omega}:=$ $e^{(r+1) \sigma} \omega$ на римановом многообразии $(M, \bar{g})$, и вследствие этого базис векторного пространства конформно киллинговых $r$-форм многообразия $(M, \bar{g})$ будет состоять из базисных нормированных $r$-форм пространства $T^{r}(M, \mathbb{R})$. На этом основании делаем вывод, что числа $t_{r}=\operatorname{dim} T^{r}(M, \mathbb{R})$ конформно инвариантны.

С помощью оператора Ходжа

$$
*: C^{\infty} \Lambda^{r} M \cong C^{\infty} \Lambda^{n-r} M
$$

(см. [1; с. 52]) был установлен изоморфизм пространств $*: T^{r}(M, \mathbb{R}) \cong T^{n-r}(M, \mathbb{R})$ (см. [2; с. 46], [9]), который позволяет теперь сформулировать свойство двойственности $t_{r}=t_{n-r}$.

Киллинговы $r$-формы, $1 \leqslant r \leqslant n-1$, образующие векторное пространство $K^{r}(M, \mathbb{R})$, на замкнутом ориентированном римановом многообразии $(M, g)$ исчерпывают $\operatorname{Ker} D^{*} D \cap \operatorname{Ker} d^{*}$ (см. [6]) и вследствие этого выполняются равенства $k_{r}=$ $\operatorname{dim} K^{r}(M, \mathbb{R})=\operatorname{dim}_{\mathbb{R}}\left(\operatorname{Ker} D^{*} D \cap \operatorname{Ker} d^{*}\right)<\infty$.

Введем в рассмотрение проективный диффеоморфизм $f:(M, g) \rightarrow(\bar{M}, \bar{g})$ как отображение, которое переводит геодезические многообразия $(M, g)$ в геодезические многообразия $(\bar{M}, \bar{g})$. Заметим, что для проективного диффеоморфизма $f$ отображение $f^{-1}:(\bar{M}, \bar{g}) \rightarrow(M, g)$ также будет проективным диффеоморфизмом.

Необходимое для доказательства теоремы свойство проективного диффеоморфизма описывает доказанная в работе [3] следующая

Лемма 2. Пусть $f:(M, g) \rightarrow(\bar{M}, \bar{g})$ - проективный диффеоморфизм $n$-мерных римановых многообразий $u \omega-$ киллинговая $r$-борма, $1 \leqslant r \leqslant n-1$, на многообразии $(M, g)$. Тогда $r$-форма $\widetilde{\omega}$ на многообразии $(\bar{M}, \bar{g})$ такая, что

$$
f^{*} \widetilde{\omega}=e^{(p+1) \psi} \omega \quad \text { для } \quad \psi=\frac{\ln (\operatorname{det} \bar{g} \operatorname{det} g)}{2(n+1)}
$$

будет киллинговой.

На основании данного утверждения заключаем, что при проективном преобразовании метрики $g \rightarrow \bar{g}$ киллинговы $r$-формы $\omega$ многообразия $(M, g)$ породят киллинговы $r$-формы $\bar{\omega}:=e^{(r+1) \psi} \omega$ риманова многообразия $(M, \bar{g})$. В результате чего базис векторного пространства киллинговых $r$-форм многообразия $(M, \bar{g})$ будет состоять из базисных нормированных $r$-форм пространства $K^{r}(M, \mathbb{R})$. На этом основании делаем вывод, что числа $k_{r}$ проективно инвариантны.

С другой стороны, $r$-формы, $1 \leqslant r \leqslant n-1$, образующие векторное пространство $P^{r}(M, \mathbb{R})$, исчерпывают $\operatorname{Ker} D^{*} D \cap \operatorname{Ker} d\left(\right.$ см. [6]), и поэтому $p_{r}=\operatorname{dim} P^{r}(M, \mathbb{R})=$ $\operatorname{dim}_{\mathbb{R}}\left(\operatorname{Ker} D^{*} D \cap \operatorname{Ker} d\right)<\infty$.

Равенство $k_{r}=p_{n-r}$, выражающее двойственность, следует из установленного ранее изоморфизма $*: K^{r}(M, \mathbb{R}) \cong P^{n-r}(M, \mathbb{R})$ (см. [2], [5]). Поскольку при этом числа $k_{r}$ проективно инвариантны, то будут проективно инвариантны и числа $p_{r}$. Теорема 1 доказана. 
Лемма 3. На п-мерном плоском римановом многообразии $(M, g)$ каждая точка обладает такой окрестностъю с локальной системой координат $x^{1}, \ldots, x^{n}$, 8 которой произвольная конформно киллинговая $r$-форма $\omega, 1 \leqslant r \leqslant n-1$, имеет локальные компоненты

$$
\omega_{i_{1} \ldots i_{r}}=A_{k j i_{1} \ldots i_{r}} x^{k} x^{j}+B_{j i_{1} \ldots i_{r}} x^{j}+C_{i_{1} \ldots i_{r}} .
$$

При этом коэфбициенты $A_{k j i_{1} \ldots i_{r}}, B_{j i_{1} \ldots i_{r}} u C_{i_{1} \ldots i_{r}}$, стоящие в правых частях равенств, являются постоянными, кососимметричными по индексам $i_{1}, \ldots, i_{r}$ и подчиняются следующим условиям:

$$
\begin{gathered}
A_{k j i i_{2} \ldots i_{r}}+A_{k i j i_{2} \ldots i_{r}}=2 A_{k i_{2} \ldots i_{r}} g_{i j}-\sum_{a=2}^{r}(-1)^{a}\left(A_{k i i_{2} \ldots \hat{i}_{a} \ldots i_{r}} g_{j i_{a}}+A_{k j i_{2} \ldots \hat{i}_{a} \ldots i_{r}} g_{j i_{a}}\right), \\
B_{j i i_{2} \ldots i_{r}}+B_{i j i_{2} \ldots i_{r}}=2 B_{i_{2} \ldots i_{r}} g_{i j}-\sum_{a=2}^{r}(-1)^{a}\left(B_{i i_{2} \ldots \hat{i}_{a} \ldots i_{r}} g_{j i_{a}}+B_{j i_{2} \ldots \hat{i}_{a} \ldots i_{r}} g_{j i_{a}}\right)
\end{gathered}
$$

$\partial \Omega я$

$$
A_{k i_{2} \ldots i_{r}}=\frac{1}{n-p+1} g^{j l} A_{k j l i_{2} \ldots i_{r}}, \quad B_{i_{2} \ldots i_{r}}=\frac{1}{n-p+1} g^{j l} B_{j l i_{2} \ldots i_{r}} .
$$

ДокАЗАТЕЛЬСтво. Пусть $(M, g)$ - плоское риманово многообразие размерности $n \geqslant 2$; по определению каждая его точка $x$ имеет окрестность $U$, изометричную открытому подмножеству в $\mathbb{R}^{n}$. В произвольной декартовой системе координат $x^{1}, \ldots, x^{n}$ окрестности $U$ локальные компоненты $g_{i j}$ метрического тензора $g$ становятся постоянными величинами, а символы Кристоффеля связности Леви-Чивиты $\nabla$ обращаются в нуль. Поэтому в $U$ ковариантное дифференцирование $\nabla_{j}:=\nabla_{\partial_{j}}$ совпадет с частным $\partial_{j}=\partial / \partial x^{j}$, в результате чего уравнения, определяющие конформно киллинговые $r$-формы (см. [10]), предстают в виде

$$
\partial_{j} \omega_{i i_{2} \ldots i_{r}}+\partial_{i} \omega_{j i_{2} \ldots i_{r}}=2 \theta_{i_{2} \ldots i_{r}} g_{i j}-\sum_{a=2}^{r}(-1)^{a}\left(\theta_{i i_{2} \ldots \hat{i}_{a} \ldots i_{r}} g_{j i_{a}}+\theta_{j i_{2} \ldots \hat{i}_{a} \ldots i_{r}} g_{i i_{a}}\right)
$$

для локальных компонент $\omega_{i_{1} i_{2} \ldots i_{r}} r$-формы $\omega$ в окрестности $U$ и компонент $\theta_{i_{2} \ldots i_{r}}=$ $(n-r+1)^{-1} g^{i j} \partial_{i} \omega_{j i_{2} \ldots i_{r}}$, которые, в свою очередь, удовлетворяют уравнениям (см. там же)

$$
\partial_{j} \theta_{i_{2} i_{3} \ldots i_{r}}+\partial_{i_{2}} \theta_{j i_{3} \ldots i_{r}}=0 .
$$

Поскольку интегралы уравнений (6) имеют вид $\theta_{i_{2} \ldots i_{r}}=A_{k i_{2} \ldots i_{r}} x^{r}+B_{i_{2} \ldots i_{r}}$ для любых кососимметричных по всем своим индексам постоянным $A_{i_{1} i_{2} \ldots i_{r}}$ и $B_{i_{2} \ldots i_{r}}$ (см. [5]), то из (5) выводим следующие уравнения:

$$
\begin{aligned}
\partial_{k} \partial_{j} \omega_{i i_{2} \ldots i_{r}}= & A_{k i_{2} \ldots i_{r}} g_{j i}+A_{j i_{2} \ldots i_{r}} g_{k i}-A_{i i_{2} \ldots i_{r}} g_{k j} \\
+ & 2^{-1} \sum_{a=2}^{r}(-1)^{a}\left(A_{i k i_{2} \ldots \hat{i}_{a} \ldots i_{r}} g_{j i_{a}}+A_{i j i_{2} \ldots \hat{i}_{a} \ldots i_{r}} g_{k i_{a}}\right. \\
& \left.\quad-A_{k j i_{2} \ldots \hat{i}_{a} \ldots i_{r}} g_{i i_{a}}-A_{j i i_{2} \ldots \hat{i}_{a} \ldots i_{r}} g_{k i_{a}}-A_{j k i_{2} \ldots \hat{i}_{a} \ldots i_{r}} g_{i i_{a}}\right),
\end{aligned}
$$

а потому $\partial_{l} \partial_{k} \partial_{j} \omega_{i i_{2} \ldots i_{r}=0}$. Последнее означает, что локальные компоненты конформно киллинговой $r$-формы $\omega$ имеют вид (4). Условия на участвующие в (4) коэффициенты появляются после подстановки этих компонент в уравнения (5). Лемма 3 доказана. 
Доказательство теоремы 2. Риманово многообразие $(M, g)$ называется конформно плоским (см. [1; с. 86]), если у любой его точки $x \in M$ существует такие окрестность $U$ и функция $\sigma$ на $U$, что многообразие $(\bar{M}, \bar{g})$ для $\bar{M}=U$ и $\bar{g}=e^{2 \sigma} g$ является плоским римановым многообразием. На плоском многообразии $(\bar{M}, \bar{g})$, как это было доказано в лемме 3 , конформно киллинговая $r$-форма $\bar{\omega}$ имеет локальные компоненты

$$
\bar{\omega}_{i_{1} \ldots i_{r}}=A_{k j i_{1} \ldots i_{r}} x^{k} x^{j}+B_{j i_{1} \ldots i_{r}} x^{j}+C_{i_{1} \ldots i_{r}} .
$$

Тогда при поточечно конформном преобразовании метрики $g=e^{2 \sigma^{\prime}} \bar{g}$ для функции $\sigma^{\prime}=-\sigma$ форма $\widetilde{\omega}=e^{(r+1) \sigma^{\prime}} \bar{\omega}$ будет согласно лемме 1 конформно киллинговой. Теорема 2 доказана.

В заключение заметим, что проведенные доказательства леммы 1 и теоремы 2 без ограничения общности могут быть перенесены на псевдоримановы многообразия, а потому будет справедливым наше замечание о возможности использования полученного результата в теоретической физике.

\section{СПИСОК ЦИТИРОВАННОЙ ЛИТЕРАТУРЫ}

[1] А. Бессе, Многообразия Эйнштейна, I, Мир, М., 1990.

[2] С.Е. Степанов, “Теоремы исчезновения в аффинной, римановой и лоренцевой геометриях", Фундамент. и прикл. матем., 11:1 (2005), 35-84.

[3] С. Е. Степанов, "О тензоре Киллинга-Яно”, ТМФ, 134:3 (2003), 382-387.

[4] Р. Нарасимхан, Анализ на действительных и комплексных многообразиях, Мир, М., 1971.

[5] S. E. Stepanov, "On conformal Killing 2-form of the electromagnetic field", J. Geom. Phys., 33:3-4 (2000), 191-209.

[6] С. Е. Степанов, "Новый сильный лапласиан на дифференциальных формах", Mатем. заметки, 76:3 (2004), 452-458.

[7] T. Branson, "Stein-Weiss operators and ellipticity", J. Funct. Anal., 151:2 (1997), 334383.

[8] Р. Пале, Семинар по теореме Атъи-Зингера об индексе, Мир, М., 1970.

[9] M. Kora, "On conformal Killing forms and the proper space of $\Delta$ for $p$-forms", Math. J. Okayama Univ., 22 (1980), 195-204.

[10] T. Kashiwada, "On conformal Killing tensor", Nat. Sci. Rep. Ochanomizu Univ., 19 (1968), 67-74.

\section{С. Е. Степанов}

Владимирский государственный педагогический университет

E-mail: stepanov@vtsnet.ru
Поступило

26.09.2005

Исправленный вариант

03.05.2006 